\title{
PHASE-DEPENDENT KNEE MODEL IDENTIFICATION IN GAIT MOTION USING AN ACTIVE KNEE REHABILITATION ORTHOTIC DEVICE
}

\author{
Ludovic Saint-Bauzel, Ozer Unluhisarcikli Student Member, IEEE, and Constantinos Mavroidis, \\ Member, IEEE
}

\begin{abstract}
This paper presents a new approach in modeling human motions using robotic rehabilitation devices. A phase dependent identification method is proposed that is based on applying a Gaussian white noise around the knee joint through the use of a rehabilitation robotic exoskeleton. The input-output relationship is analyzed and the concept to represent the walking motion as a phase-dependent action is described. The method takes advantage of the time independent property of Gaussian noise and shows great accuracy in modeling human limb motions when compared to classical (i.e. global) models. The new modeling method for human limb motions is important in the control and planning of rehabilitation robotic devices and in evaluating human biomechanics using a portable gait measuring device.
\end{abstract}

\section{INTRODUCTION}

Rehabilitation robotics that has seen great growth during the last years can be classified into end-effector devices and wearable exoskeleton devices. The end effector design approach is both applied on upper limb (e.g. MIT Manus [1],Handle Device[2])) and on lower limb rehabilitation (Haptic Walker [3]). Similarly, exoskeleton devices could be used on upper limb (Able[4], 2RobotArm[5]) and lower limb (Lokomat[6],PAM-POGO[7], KneeDevice[8]). In both types of rehabilitation devices the controllers are similar. However due to the additional safety concerns when wearing a an exoskeleton, its controller should guarantee safe operation of the device..

In general, the main control aims of a rehabilitation robotic devices are that the controller should be able to a) implement accurately the prescribed rehabilitation treatment protocol and b) eliminate unwanted (e.g. hazardous) effects on the subject. In order for the controller to be able to achieve these aims, it should be able to take into account the changing dynamics of the human body and its environment using a dynamic model.

One way to develop a dynamic model of the human body interacting with its

This work was supported in part by the U.S. National Science Foundation under Grant CBET-0828772. Any opinions, findings, conclusions, or recommendations expressed in this publication are those of the authors and do not necessarily reflect the views of the National Science Foundation.

L. Saint-Bauzel is with the Université Pierre et Marie Curie-UPMC "Institut des Systèmes Intelligents etde Robotique" ISIR, Paris, F-75014 France (corresponding author to provide phone: (+33)144-276-210; fax: (+33)144-275-145; e-mail: saintbauzel@isir.upmc.fr).

O. Unluhisarcikli and C. Mavroidis are with the Department of Mechanical and Industrial Engineering, Northeastern University, Boston MA 02115 USA (phone: 617-373-4121; fax: 617-3732921; e-mail: mavro@coe.neu.edu).

Corresponding authors: L. Saint-Bauzel and C. Mavroidis 
environment for use in the control of a rehabilitation device, is to use a global model that takes into account the whole body. However, This global modeling leads to a very large number of parameters to be identified using experimental data and a complex identification process [4,5 or 6] It is also computationally very expensive that makes its real time implementation on the device almost impossible. [14], [15] and [16]. But this bio-mechanical models that aim to understand and present a realistic model of the joint. In our case we want to represent properties during defined motion. As describe below the walking lead to non-linearities when the foot impact the ground, many unwanted motion are appearing because of the robot attachment. So the issue is that one don't know if the knee properties while walking motion can be presented as a linear parameter variable system. So this paper will evaluate if it is accurate to represent the knee mechanical properties as a LPV system and present a ensemble data method to identify the parameters. It will be applied on knee motion equipped of ANDROS Device that present the property to be a one DOF exoskeleton and is therefor a good experimental tool to apply the method.

\section{System Overview - ANDROS V1}

ANdROS V1 is designed as a wearable and portable assistive tool for gait rehabilitation and monitoring. The exoskeleton frame consists of two braces: an actuated brace attached to the impaired leg via rigid straps, and a sensorized brace attached to the healthy leg via elastic straps (Figures 1 \& 2). The power and torque specifications of ANdROS were determined based on the reference trajectories obtained from tests conducted on 70 adult healthy subjects at Spaulding Rehabilitation Hospital in Boston, MA. The specifications obtained from these tests are summarized in Table 1.

The actuated brace is driven by a brushless DC motor (MCG IB23000-E1) coupled to a gear-

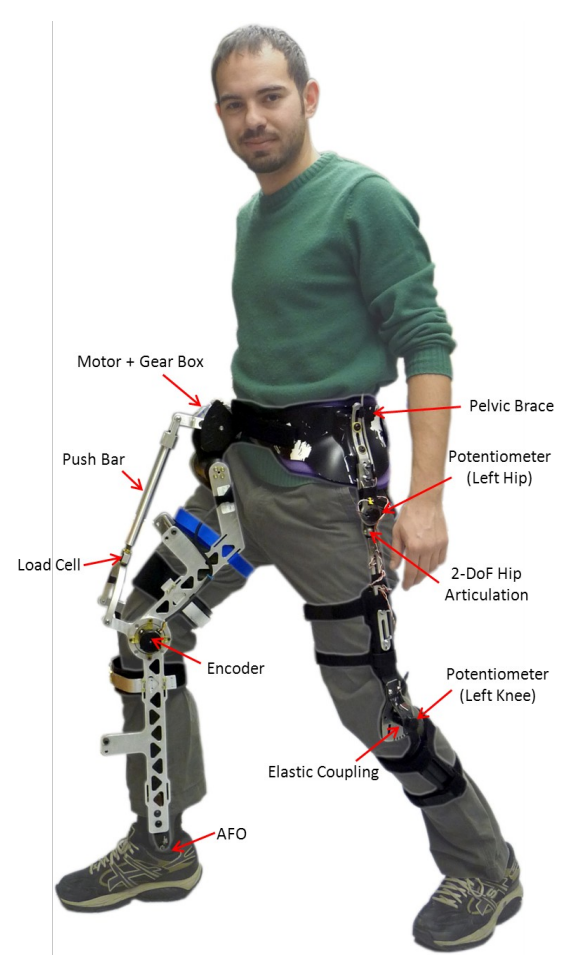
box (Anaheim Automation GBPH-0602-NP-040, 40:1 gear Figure 1: ANdROS V1: The new Active Knee ratio) which is located next to Rehabilitation Orthotic System. the pelvis, to reduce the moving 
mass and keep the center of mass (CM) of the exoskeleton close to the wearer's $\mathrm{CM}$. The motor has been selected to provide at least $50 \%$ assistance. The peak torque at the gearbox output is $22.88 \mathrm{Nm}$ and rated power is $179 \mathrm{~W}$. The torque generated by the motor is transferred to the knee joint by means of a push bar that features a quick disengagement mechanism. By disengaging the lower part of the exoskeleton from the power train, easy adjustment during donning/doffing is possible. The push bar mechanism of ANdROS is basically a four bar linkage. Kinematic analysis has been carried out to convert the torque at the mo-

TABLE 1: TECHNICAL SPECIFICATIONS

\begin{tabular}{lcc}
\hline & $10 \%$ of Gait Cycle & $90 \%$ of Gait Cycle \\
\hline Torque $^{\mathrm{a}}(\mathrm{Nm})$ & 54 (peak) & 27 \\
Velocity $(\mathrm{rad} / \mathrm{sec})$ & 2 & 7 (peak) \\
Power $(\mathrm{W})$ & 108 & 189 \\
\hline \hline
\end{tabular}

tor shaft to the torque at the knee, but has been excluded herein due to space limitations.

A load cell at the end of the push bar measures the interaction forces. The load cell is located proximal to the knee joint to maximize the amount of mass between the motor and itself, whose apparent inertia is reduced by force-feedback. An incremental encoder and a rotary potentiometer are placed on either side of the knee joint, where the potentiometer is used to initialize the incremental encoder's position. The rotary encoder provides high accuracy $\left(0.088^{\circ}\right)$ and noise free measurement of the knee angle, which are critical properties in calculating digital derivative for velocity estimation.

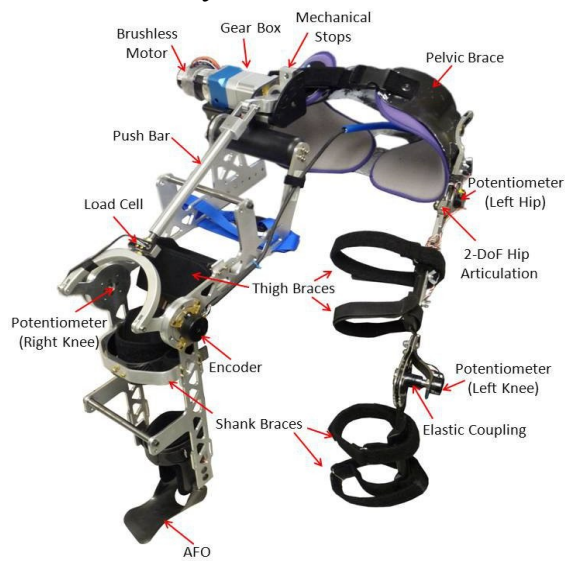

Figure 2: Close-Up View of ANdROS V1 and its Components.

An exoskeleton should be attached to the body so that there is minimal play (migration) with respect to the human tissue, yet still be comfortable to wear. The actuated brace of ANdROS is attached to the user's body at four locations (i.e. hip, thigh, shank, and ankle) to prevent the migration effect. The weight of the exoskeleton and the user is transferred to the ground through an AFO. The 
symmetrical structure of the actuated brace applies torque to the knee from both medial and lateral sides of the leg, thus eliminating the twisting effect which would occur in a single-sided design.

The brace worn on the unimpaired leg measures the knee and hip flexion/extension angles via high precision potentiometers.

\section{Models}

\subsection{Phases of Gait Cycle}

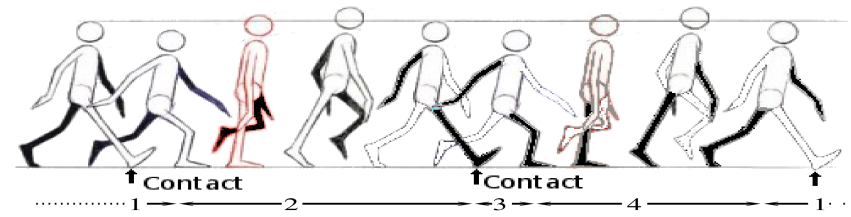

Figure 3: One Stance Cycle Schematic Representation.

Figure 3 shows the four phases in a gait cycle. The stance is supposed to begin when the foot is close to leave the ground. During Phase- 1 (from 0 to $20 \%$ of the gait cycle) one can see that the right foot is in the air. Then Phase- 2 is a high torque phase corresponding to the ground hitting pick (between 20 to $40 \%$ of the gait cycle). Then a second opposite pick is observed corresponding to the main knee action to move the other leg in Phase-3 (40-60\% of the gait cycle). The last phase, Phase-4, is more or less a constant part of the walking motion for the knee. It is when the leg is straight and turns around the ankle while the other foot is in the air and goes to the front of the subject.

\subsection{Mechanical Models of the Knee}

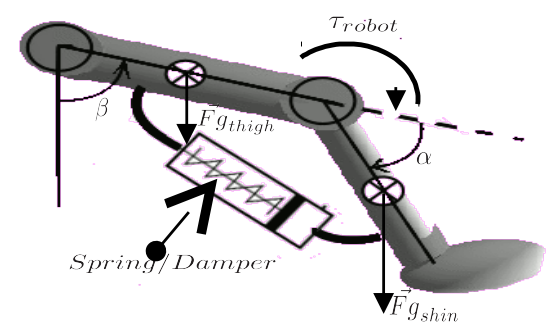

Figure 4: Mechanical Impedance Representation of the Leg

The biomechanical model of the knee shown in Figure 4 leads to Equation (1).

$$
\tau_{\text {muscle }}+\tau_{\text {robot }}+\tau_{\text {gravity }}=I \ddot{\omega}
$$

where $\tau_{\text {muscle }}, \tau_{\text {robot }}$ and $\tau_{\text {gravity }}$ are respectively the torque coming from the leg muscle, the robot actuator and the gravity on the leg, $I$ is the inertia of the 
system. But one hypothesis is that the actuators are not perfect so they have some impedance (where $k$ represents spring parameter and $b$ stands for damper actions) in the motion. $U$ is the initial torque.

Consequently $\tau_{\text {robot }}$ and $\tau_{\text {muscle }}$ will be written a impedance torques :

$$
\begin{aligned}
& \tau_{\text {muscle }}=U_{\text {muscle }}+k_{\text {muscle }} \Delta \alpha+b_{\text {muscle }} \Delta \dot{\alpha} \\
& \tau_{\text {robot }}=U_{\text {rob }}+k_{\text {rob }} \Delta U+b_{\text {rob }} \Delta \dot{U}
\end{aligned}
$$

So the previous equation becomes :

$$
\begin{gathered}
U_{\text {muscle }(t)}+k_{\text {muscle }(t)} \Delta \alpha+b_{\text {muscle }(t)} \Delta \dot{\alpha} \\
+U_{\text {rob }}+k_{\text {rob }} \Delta U_{\text {rob }}+b_{\text {rob }} \Delta U_{\text {rob }} \\
+\tau_{\text {gravity }}+\Phi_{w}=\left(I_{\text {rob }}+I_{\text {shin }}\right) \ddot{\omega}
\end{gathered}
$$

where $\omega$ is the global orientation of the shin, $\alpha$ is the angle between the shin and the thigh. And $I$ is decompose of inertia property of the shin $I_{s h i n}$ and inertia of the robot $I_{r o b}$. One can notice that these equation are representing the dynamics of the leg without external forces coming from the ground during the walking motion. As a reminder, time varying parameters are identified in this equation. In this paper one would like to represent this ground action as a part of time varying impedance. So as a time varying impedance, it has been added to the previous equation and is called $\Phi_{w}$.

The idea of this work is to represent walking motion as a time varying impedance. So it seems that we may have to represent $\Phi_{w}$ as a function of time $t$ and $\alpha$. The problem is that a big part of walking motion is done with $\alpha$ close to a blocked position (during the swing of the other Leg). And the action of the robot could be useful during this motion. So the proposed solution is to include Hip angle $(\beta)$ in the time varying model of the walking motion.

So the time varying of dynamics of walking can be presented as follow :

$$
\Phi_{w(t, \alpha, \beta)}=U_{(t)}+k_{w 1(t)} \alpha+b_{w 1(t)} \dot{\alpha}+k_{w 2(t)} \beta+b_{w 2(t)} \dot{\beta}
$$

where $U(t)$ will be considered as a time varying wanted torque.

Having an identification of the human joint mechanical parameters is not the aim of this paper. The aim is to have a model of the leg connected to the robot. Consequently knowing the $k_{m u s c l e(t)}$ and $b_{\text {muscle }(t)}$ parameters for themself is not relevant. In this paper, it will be added in (6) as follow :

$$
\Phi_{w m(t, \alpha, \beta)}=\Phi_{w(t, \alpha, \beta)}+k_{\text {muscle }(t)} \alpha+b_{\text {muscle }(t)} \dot{\alpha}
$$

Consequently, the parameters that are dependent to $\alpha$ and its differentiates can be aggregated and will be called :

$$
k_{w m 1(t)}=k_{w 1(t)}+k_{m u s c l e(t)}(7), b_{w m 1(t)}=b_{w 1(t)}+b_{m u s c l e(t)}(8)
$$

\subsection{Phase-dependent representation}

In this paper, the idea is to take advantage of periodicity of walking motion. So an hypothesis of this work is that there is some periodicity in the properties of the leg between each stance. Thanks to this property, the time varying 
parameters will be considered as phase-dependent parameter. So one can introduce a new parameter $\Omega$ that represents the phase of the motion. This parameter is only a position of the phase. It is a real scalar value between 0 and 1 that represents a percent of the phase.

The difficulty is that this numerical representations are not representing the proximity between a parameter close to 0 and a parameter close to 1 . In order to obtain a realistic relation, it has been decided to use a lookup table that represent a piece of data centered on the percent presented by $\Omega$ and a time window of $W$. As for example, $k_{w m 1(\Omega, W)}$ is computed with every data at time $t_{\Omega} \pm W * \frac{T_{\text {stance }}}{2}$ where $t_{\Omega}$ are every moment that are in $\Omega$ position of the stance motion, $T_{\text {stance }}$ is the duration of the stance and $W$ is a percent of this duration. Consequently, $k_{w m 1(0, W)}=k_{w m 1(1, W)}$ and continuity is kept. Another property of this notation is one can represent a fixed in time parameter as robot parameters for example:

$k_{r o b}=k_{r o b(x, 1)}, b_{r o b}=b_{r o b(x, 1)}, \forall x$

From equation (1) with impedance parameters ( $k$ for spring action, $b$ for damper action parameter), Inertia matrices decomposed by their origin $\left(I_{\text {robot }}+I_{\text {shin }}\right)$, the system can therefore be rewritten as follow:

$$
\begin{aligned}
& U_{\text {muscle }(\Omega)}+U_{\text {rob }(t)}+k_{\text {rob }} \Delta U_{\text {rob }}+b_{\text {rob }} \Delta U_{\text {rob }} \\
& +k_{w m 1(\Omega, W)} \Delta \alpha+b_{w m 1(\Omega, W)} \Delta \dot{\alpha}+k_{w 2(\Omega, W)} \Delta \beta \\
& +b_{w 2(\Omega, W)}+\tau_{\text {gravity }}=\left(I_{\text {robot }}+I_{\text {shin }}\right) \ddot{\omega}
\end{aligned}
$$

where all the $\Omega$ dependent parameters, $k$ and $b$ robot parameters and $\left(I_{\text {robot }}+I_{\text {shin }}\right)$ are unknown and are the parameters that are aimed to be identified in this paper.

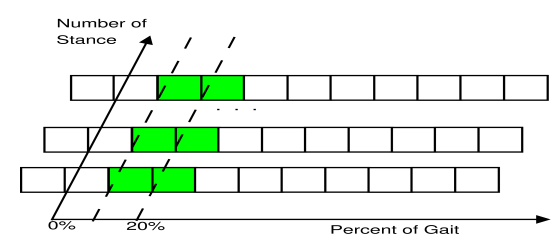

Figure $5: \operatorname{data}(\Omega, W)$ with $\Omega=0.2$ and $W=0.2$
The idea behind this variable model is to take a small set of data around the working point and to find the best model parameters by converging to the local minimum. The data selection is phase $d$ dependent and is collected as explained before for the parameters and as presented in Figure 5.

This approach is also a way to reduce inter-trial variations, and as far as time evolutions is not modified it is respecting dynamics properties of the motion.

\section{Results \& Discussion}

\subsection{Experimental setup}

The comparison is done between a model Model $_{w i(-, 1)}$ with constant parameters and the model with phase varying parameters is called 
Model $_{w i(\Omega, 0.2)}$ with $i \in[m 1,2]$ and because the evaluated window size is $20 \%$ of stance.

\subsection{Results}

If one compares the proposed models as they estimate the knee torque applied by the human when walking with AKROD, we can observe that the phase varying parameter model is closer to the constant parameter model.

As presented in Fig. 6, the black line that represent the phase model torque evaluation seems closer to grey line than green dashed line, the most relevant parts are around sample 200. and around the sample 100.

A better way to evaluate the accuracy of the phase varying model is to look at the measured quadratic error along the stance as it is done in Fig. 7. According to this graphic, the phase varying model is always under the constant model, It

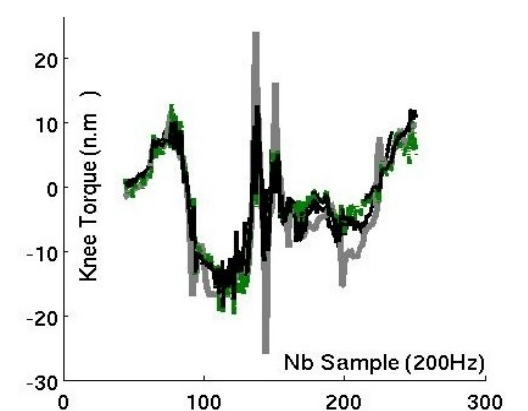

Figure 6: Model(-,1) (green and dashed line), Model $(\Omega, 0.2)$ (Black line), and Measured data(grey line) of Knee Torque during one stance.

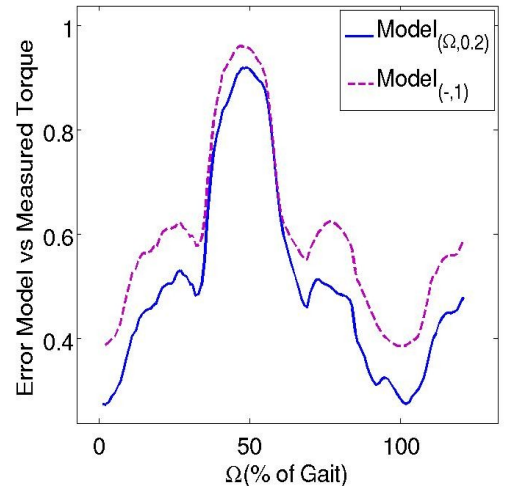

Figure 7: Comparison Phase dependent model Model $\left._{(\Omega, W)}\right)$ vs global model Model $\left._{(-, 1)}\right)$ error along motion

means that the errors between model predicted torque and measured torque.

\subsection{Discussion}

This study shows a phase-dependent model that fits to a human leg with good accuracy. The methodology based on random noise is an interesting approach in obtaining a dynamic model. However, as explained in the introduction, one of the goals of this work is to create a model of the patient. Obviously, introducing random noise to the patient while he/she is in motion can be a problem. Therefore, one limitation of this method is the patient being subjected to perturbations as he/she walks during the identification process.

This method is also interesting in showing the different fields of accuracy of the proposed model. Since this is a phase dependent algorithm, it is possible to propose more accurate models in some parts of the motion.

It should be noted that this study is conducted on a single subject. This is a proof of concept that must be confirmed with statistical analysis on multiple 
subjects. However, this larger study will have to pay attention to the subjectdependency of this method.

\section{References}

[1] Hogan, N.; Krebs, H.; Charnnarong, J.; Srikrishna, P. \& Sharon, A.,Robot and Human Communication, 1992. Proceedings., IEEE International Workshop on : 161 -165(1992).

[2] Khanicheh, A.; Mintzopoulos, D.; Weinberg, B.; Tzika, A. \& Mavroidis, C.,Rehabilitation Robotics, 2007. ICORR 2007. IEEE 10th International Conference on : 883 -889(2007).

[3] Schmidt, H.; Hesse, S.; Bernhardt, R. \& Krüger, J.,ACM Transactions on Applied Perception 2 : 166-180(2005).

[4] Garrec, P.; Friconneau, J.; Measson, Y. \& Perrot, Y.,Intelligent Robots and Systems, 2008. IROS 2008. IEEE/RSJ International Conference on : 1483 $-1488(2008)$.

[5] Culmer, P.; Jackson, A.; Richardson, R.; Bhakta, B.; Levesley, M. \& Cozens, A.,Rehabilitation Robotics, 2005. ICORR 2005. 9th International Conference on : $61-65(2005)$.

[6] Bernhardt, M.; Frey, M.; Colombo, G. \& Riener, R.,Rehabilitation Robotics, 2005. ICORR 2005. 9th International Conference on : 536-539(2005).

[7] Aoyagi, D.; Ichinose, W.; Harkema, S.; Reinkensmeyer, D. \& Bobrow, J., 15 : 387 -400(2007).

[8] Weinberg, B.; Nikitczuk, J.; Patel, S.; Patritti, B.; Mavroidis, C.; Bonato, P. \& Canavan, P.,Robotics and Automation, 2007 IEEE International Conference on : $4126-4133(2007)$.

[9] Jarrasse, N.; Paik, J.; Pasqui, V. \& Morel, G.,Robotics and Automation, 2008. ICRA 2008. IEEE International Conference on : 2134 -2139(2008).

[10] Zhang, L.-Q.; Nuber, G.; Butler, J.; Bowen, M. \& Rymer, W. Z.,Journal of Biomechanics $31: 71$ - 76(1997).

[11] Kiss, R.; Kocsis, L. \& Knoll, Z.,Med.Eng Phys. 26 : 611-620(2004).

[12] Sok, K. W.; Kim, M. \& Lee, J.,SIGGRAPH '07: ACM SIGGRAPH 2007 papers : 107(2007).

[13] Sasaki, O.; Gagey, P.; Ouaknine, M.; Martinerie, J. andVan Quyen, M.; Toupet, M. \& L'Heritier, A.,Neuroscience Research 41 : 185-192(2001).

[14] Xu, Y. \& Hollerbach, J.,Biomedical Engineering, IEEE Transactions on 45 : $1051-1060(1998)$.

[15] Xu, Y. \& Hollerbach, J.,Biomedical Engineering, IEEE Transactions on 46 : 409 -419(1999).

[16] Konczak, J.; Brommann, K. \& Kalveram, K.,Motor Control 3(4) : 394413(1999). 